\title{
Détermination des normes de tolérance thermophysiologique pour le travail en installation nucléaire de base en tenue TIVA
}

\author{
P. CASANOVA ${ }^{1}$, J-L. DARET ${ }^{1}$, Y. BESNARD ${ }^{2}$, N. CLERC ${ }^{2}$, \\ G. SAVOUREY ${ }^{2}$, J-C. LAUNAY ${ }^{2}$
}

(Manuscrit reçu le 4 janvier 2011, accepté le 17 février 2011)

RÉSUMÉ Cette étude a eu pour but de déterminer la durée du port de la tenue ventilée TIVA au cours d'un exercice physique à la chaleur en fonction de l'atteinte de seuils physiologiques de sécurité. Cinq sujets volontaires équipés de la tenue ventilée TIVA ont effectué un exercice sur tapis roulant $(3,5 \mathrm{~km} / \mathrm{h}, 3 \%$ de pente) en chambre thermoclimatique à 25,35 et $45{ }^{\circ} \mathrm{C}$ d'une durée maximale de $2 \mathrm{~h}$. La fréquence cardiaque, la température rectale (Tre) et la température cutanée moyenne ( $\overline{\mathbf{T}} \mathrm{sk}$ ) ont été mesurées en continu. La déshydratation a été calculée par la perte de poids au cours de l'exercice. À $25^{\circ} \mathrm{C}$, il n'y a pas d'atteinte des seuils thermiques ou cardiovasculaires mais il y a un risque de déshydratation si l'exercice se prolonge. Par contre pour les tests à 35 et $45^{\circ} \mathrm{C}$, la durée limite d'exposition (DLE) est limitée, pour le seuil de Tre à $39^{\circ} \mathrm{C}$, à $97 \pm 29$ et $69 \pm 28 \mathrm{~min}$, respectivement. Pour l'atteinte du seuil d'augmentation de la Tre de $+1,5^{\circ} \mathrm{C}$, les DLE sont de $84 \pm 38 \mathrm{~min}$ à $35^{\circ} \mathrm{C}$ et de $57 \pm 21$ min à $45^{\circ} \mathrm{C}$. Les conditions à $35^{\circ} \mathrm{C}$ et $45^{\circ} \mathrm{C}$ conduisent à un stockage thermique $\left(42,2 \pm 22,9 \mathrm{~W} / \mathrm{m}^{2}\right.$ et $63,7 \pm 29,4 \mathrm{~W} / \mathrm{m}^{2}$, respectivement) exposant le sujet à un risque d'accident hyperthermique auquel s'ajoute une déshydratation $(2 \%$ du poids corporel) ce qui sollicite particulièrement le système cardio-vasculaire. Ceci conduit à préconiser des durées limites d'utilisation de la tenue TIVA pour un exercice physique type dans des conditions climatiques connues (entre 25 et $45{ }^{\circ} \mathrm{C}$ ).

ABSTRACT Determination of the standards of the thermophysiological tolerance for working in basic nuclear installation in TIVA protective clothing.

The purpose of this study was to determine the duration limited exposure (DLE) for working in a contaminated zone in a basic nuclear installation using the new ventilated TIVA protective clothing. Five subjects underwent a treadmill exercise $\left(3.5 \mathrm{~km} / \mathrm{h}, 3 \%\right.$ of slope) of up to $2 \mathrm{~h}$ in a climatic chamber at 25,35 , and $45{ }^{\circ} \mathrm{C}$. Cardiac frequency, rectal temperature and skin temperature were continuously recorded. The dehydration level was measured by the loss of weight during the exercise. At $25{ }^{\circ} \mathrm{C}$, thermal and cardiovascular thresholds are not reached; the risk is dehydration if the exercise is prolonged. In contrast, the thresholds for Tre at $39{ }^{\circ} \mathrm{C}\left(97 \pm 29\right.$ and $69 \pm 28 \mathrm{~min}$ for 35 and $45^{\circ} \mathrm{C}$, respectively $)$ and for the increase in Tre $\left(+1.5^{\circ} \mathrm{C}\right)$ are reached earlier $\left(84 \pm 38 \mathrm{~min}\right.$ and $57 \pm 21 \mathrm{~min}$ at 35 and $45{ }^{\circ} \mathrm{C}$, respectively). This leads to a higher heat storage $\left(42.2 \pm 22.9 \mathrm{~W} / \mathrm{m}^{2}\right.$ and $63.7 \pm$ $29.4 \mathrm{~W} / \mathrm{m}^{2}$, at 35 and $45{ }^{\circ} \mathrm{C}$, respectively), which constitutes a higher risk of heat illnesses. The dehydration level is $2 \%$ of the weight for the tests at 35 and $45{ }^{\circ} \mathrm{C}$. As a consequence, DLE for working in a contaminated zone in a basic nuclear

AREVA NC- établissement de La Hague, service de santé au travail, 50444 Beaumont-Hague Cedex, France.

2 IRBA, Antenne La Tronche CRSSA, 24 avenue du marquis de Grésivaudan, BP 87, 38702 La Tronche Cedex, France. 
installation in environmental conditions and using the new ventilated TIVA protective clothing, as encountered in our experiments, are proposed to avoid hyperthermic accidents.

Keywords: Radiation protection / occupational exposure / guidelines / risk analysis / heat constraint

\section{Introduction}

Une Tenue d'intervention ventilée adaptable (TIVA) a été mise au point pour le travail en installation nucléaire de base (INB). C'est une tenue ventilée pressurisée à « double peau » conforme à la norme EN 1073-1. Outre sa compatibilité avec d'autres équipements de protection individuelle (harnais antichute, casque, protection individuelle contre le bruit), son originalité par rapport aux tenues existantes réside dans le choix de l'équipement de protection respiratoire constitué d'une cagoule ayant sa propre ventilation à partir d'une dérivation en interne de l'alimentation en air de la tenue (Photos 1 \& 2). Cet équipement est lui-même certifié EN 14594 (appareil de protection des voies respiratoires isolant à adduction d'air), ce qui autorise son usage en autonomie pour des interventions sur boîte à gants.

Une tenue étanche comme celles utilisées dans le nucléaire constitue une double contrainte thermique pour l'individu: d'une part son poids et son ergonomie, qui se traduisent par une augmentation de la dépense énergétique, dont $75 \%$ est transformée en chaleur du fait du faible rendement de la contraction musculaire ; d'autre part son étanchéité, qui empêche l'évaporation sudorale, donc

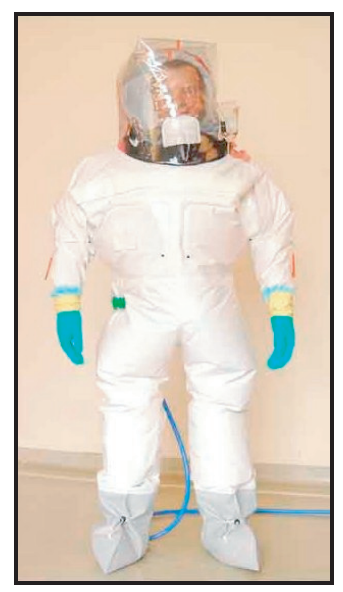

Photo 1 - Tenue TIVA.

TIVA protective clothing.

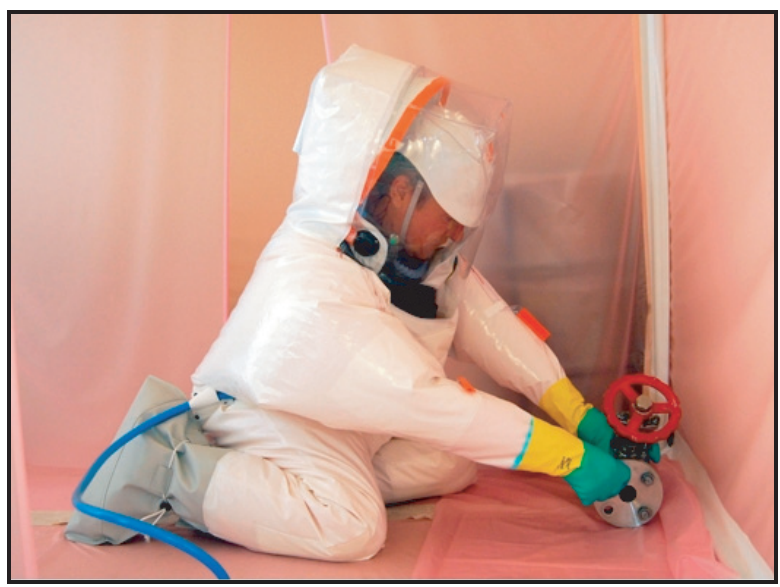

Photo 2 - Tenue TIVA en action. TIVA protective clothing in action. 
limite voire annihile la thermolyse, du fait que le volume d'air de l'espace sousvestial, compris entre la peau et la tenue, se sature rapidement en vapeur d'eau provenant de l'évaporation de la sueur. Ainsi le port d'une tenue étanche augmente le stockage de chaleur et donc, représente un facteur favorisant la survenue d'accidents médicaux liés à l'hyperthermie (coup de chaleur et déshydratation).

La TIVA est une tenue ventilée avec de l'air sec, ce qui favorise la thermolyse du fait de son important débit de renouvellement et de sa sécheresse. Ainsi la durée de son port ou durée limite d'exposition (DLE) doit être plus importante que celle d'une même tenue non ventilée : c'est ce que nous avons voulu déterminer au cours d'un exercice physique modéré effectué dans trois conditions climatiques différentes modélisant au mieux des situations habituellement rencontrées dans les chantiers de décontamination. Ces durées limites correspondent au temps nécessaire à l'atteinte de seuils physiologiques prenant en compte le risque d'accident hyperthermique et/ou la déshydratation.

\section{Matériel et méthodes}

\subsection{Les sujets}

Cinq sujets masculins volontaires (S1 à S6), spécialistes de la décontamination de sites nucléaires et âgés de 40 à 51 ans, ont été sélectionnés après examen médical par un médecin du travail. Une exploration fonctionnelle à l'exercice, avec électrocardiogramme (ECG), détermination de l'aptitude physique aérobie et de la puissance maximale aérobie, a été pratiquée. Elle a conduit à poser l'inaptitude du sujet S4 (Tab. I).

TABLEAU I

Caractéristiques biométriques des sujets. Biometric data.

\begin{tabular}{|c|c|c|c|c|c|c|c|}
\hline SUJET & S1 & S2 & S3 & S5 & S6 & Moyenne & Écart-type \\
\hline Âge (années) & 45 & 41 & 51 & 40 & 50 & 45,40 & 5,03 \\
\hline Taille (m) & 1,85 & 1,80 & 1,82 & 1,77 & 1,77 & 1,80 & 0,03 \\
\hline Poids (kg) & 86 & 89 & 75 & 68 & 73 & 78,20 & 8,93 \\
\hline Surface corporelle $\left(\mathrm{m}^{2}\right)$ & 2,09 & 2,08 & 1,95 & 1,83 & 1,89 & 1,97 & 0,11 \\
\hline PMA (W) & 980 & 980 & 980 & 820 & 1040 & 960 & 74 \\
\hline $\mathrm{VO}_{2} \max (\mathrm{L} / \mathrm{min})$ & 3,13 & 3,40 & 3,08 & 2,04 & 3,63 & 3,06 & 0,61 \\
\hline FC max (bpm) & 169 & 176 & 167 & 177 & 170 & 171,80 & 4,44 \\
\hline $\mathrm{VO}_{2} \max (\mathrm{ml} / \mathrm{min} / \mathrm{kg})$ & 36,40 & 38,20 & 41,07 & 30,00 & 49,73 & 39,08 & 7,21 \\
\hline
\end{tabular}




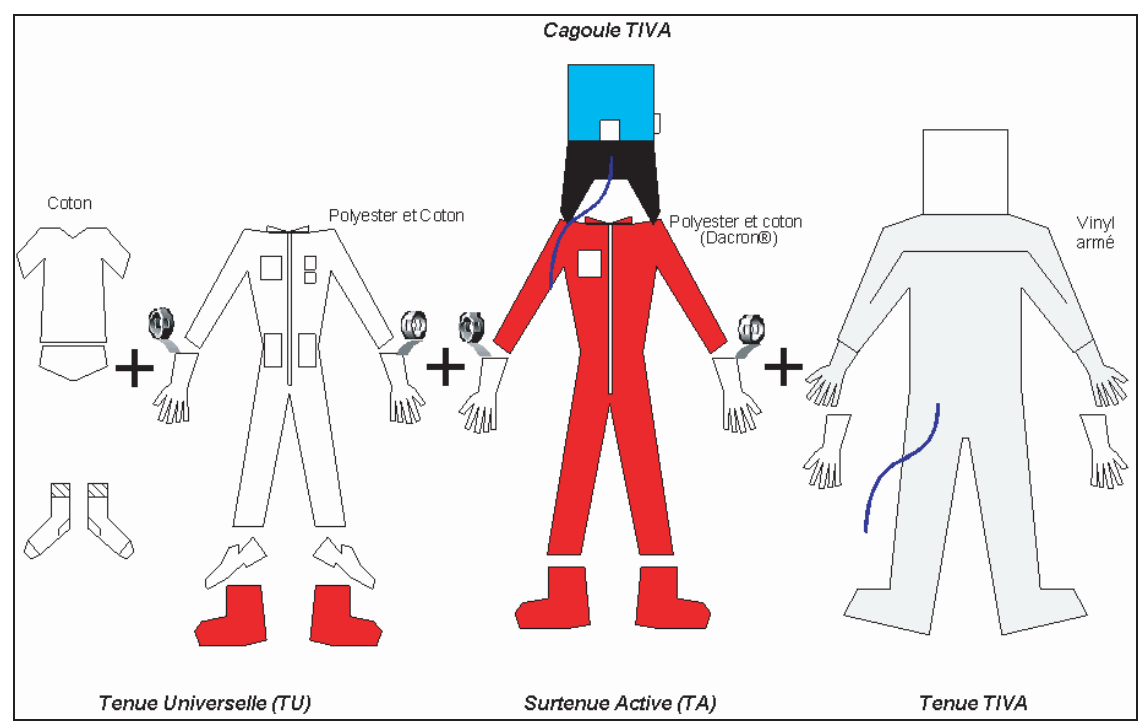

Figure 1 - Détails de l'ensemble de la tenue TIVA.

Details of TIVA protective clothing.

L'expérimentation s'est déroulée dans la chambre thermoclimatique chaude à l'Institut de recherche biomédicale des armées - Antenne La Tronche - CRSSA qui a une autorisation de lieux de recherches biomédicales sans bénéfice individuel direct et a reçu un avis favorable du Comité de protection des personnes de Grenoble le 12 septembre 2002. Les sujets ont été déclarés au fichier national des volontaires bénévoles.

\subsection{Les 3 tests de l'expérimentation}

\subsubsection{Conditions climatiques et équipement}

Chaque sujet a réalisé trois tests $\left(25,35,45{ }^{\circ} \mathrm{C}\right.$, humidité relative $=20 \%$, vent $=$ $1 \mathrm{~m} / \mathrm{s})$ en chambre thermoclimatique avec marche sur tapis roulant $(3,5 \mathrm{~km} / \mathrm{h}$, pente $3 \%$ ) portant l'équipement AREVA suivant comme décrit figure 1 :

-1 slip,

- 1 paire de chaussettes,

- 1 tee-shirt,

- 1 paire de chaussures de sécurité,

- 1 combinaison blanche (tenue universelle),

- 1 combinaison rouge (sur-tenue dite tenue active), 
- 3 paires de gants latex,

- 3 paires de sur-bottes,

plus la tenue TIVA, sa cagoule avec cartouche filtrante et système de raccordement pour la ventilation.

Compte-tenu du poids de l'équipement ( $8,2 \mathrm{~kg}$ en moyenne), la dépense énergétique estimée avec la formule de Pandolf (1977) est respectivement de 359, $372,316,309$ et $310 \mathrm{~W}$ pour les sujets $\mathrm{S} 1, \mathrm{~S} 2, \mathrm{~S} 3, \mathrm{~S} 5$ et $\mathrm{S} 6$ ce qui correspond pour ces sujets à $36,6 \%, 37,9 \%, 32,2 \%, 37,6 \%$ et $29,8 \%$ de la puissance maximale aérobie. Cette dépense énergétique est voisine de celle observée chez un travailleur effectuant un travail au marteau pneumatique comme décrit dans la norme ISO 8996, 2004 (comprise entre 235 à $360 \mathrm{~W}$ avec une moyenne à $295 \mathrm{~W}$ ).

L'ordre de passage a été randomisé et les tests ont été séparés d'au moins un jour, pour éviter au mieux les premiers effets d'une possible adaptation à la chaleur. L'étude a été réalisée en simple aveugle et s'est déroulée sur deux semaines.

L'air ventilant la tenue était de qualité médicale et fourni avec un débit constant dans la tenue de $640 \mathrm{NL} / \mathrm{mn}$, à une température de $25 \pm 2{ }^{\circ} \mathrm{C}$ et sous une pression de 6 à 8 bars.

Il n'y avait pas de réhydratation pendant le test.

\subsubsection{Les variables mesurées et calculées}

Les mesures physiologiques suivantes sont effectuées :

- températures cutanées en 10 sites permettant le calcul de la température cutanée moyenne ( $\overline{\mathrm{T}} \mathrm{sk}$ ) selon Colin et Houdas (1965) et température rectale (Tre) ; la mesure de ces températures permet de déterminer la variation de la température corporelle moyenne ( $\mathrm{Tb}$ ) et donc le stockage thermique $\mathrm{S}\left(\mathrm{W} / \mathrm{m}^{2}\right.$ de surface corporelle) par thermométrie,

- fréquence cardiaque (FC),

- saturation artérielle en oxygène $\left(\mathrm{SpO}_{2}\right)$,

- variations de poids du sujet et des équipements :

- la différence de poids du sujet équipé observé en fin d'expérimentation par rapport au poids initial permet d'avoir une estimation de la quantité de vapeur d'eau transférée au travers des équipements,

- la différence de poids du sujet nu observé en fin d'expérimentation par rapport au poids initial permet la détermination du niveau de déshydratation des sujets, 
- les variations de poids des équipements permettent de déterminer la quantité de sueur piégée dans les vêtements,

- les thermogrammes des tenues à l'aide d'une caméra infrarouge FLIR SC3000.

Des questionnaires de confort thermique (très froid : -4 ; neutre : 0 ; très chaud : +4), de fatigue (basée sur l'échelle de Borg, 1974 : de 6 : très très légère à 20 : très très pénible) et de confort de port ont été remplis à la fin de chaque test (Borg et Dahlstrom, 1962).

\subsection{Détermination des durées limites d'exposition (DLE)}

Les durées limites d'exposition (DLE) liées au stockage thermique sont déterminées pour chaque sujet aux seuils suivants (norme ISO 9886) :

- « alarme » : augmentation de la température rectale de $0,8^{\circ} \mathrm{C}$;

- « danger» : augmentation de la température rectale de $1{ }^{\circ} \mathrm{C}$;

- «extrême danger » : augmentation de la température rectale de $1,5^{\circ} \mathrm{C}$.

Le seuil « danger » de déshydratation, pour un sujet non acclimaté à la chaleur, est de $1000 \mathrm{~g} / \mathrm{h}$. Le seuil limite de fréquence cardiaque est de $185-0,65 \times$ âge (âge en année) (normes ISO 9886 et 7933).

Lorsqu'un de ces seuils n'est pas atteint et que les variables se stabilisent à des niveaux inférieurs, la durée du test est limitée à $2 \mathrm{~h}$, compte tenu de la pénibilité des conditions expérimentales.

\section{Résultats}

\subsection{Les tests $\grave{a} 25^{\circ} \mathrm{C}$}

À $25{ }^{\circ} \mathrm{C}$, la contrainte thermique est faible, les températures corporelles et la fréquence cardiaque se stabilisent en fin de tests à des niveaux acceptables (Tre = $38,3 \pm 0,4{ }^{\circ} \mathrm{C} ; \overline{\mathrm{T}} \mathrm{sk}=35,6 \pm 0.7^{\circ} \mathrm{C}$ et $\mathrm{FC}=121 \pm 14 \mathrm{bpm}$ ) traduisant un équilibre thermique avec ressenti de «confort thermique» $(0,6 \pm 0,5)$. Le stockage thermique est ainsi limité à $19,2 \pm 4,5 \mathrm{~W} / \mathrm{m}^{2}$.

De ce fait, à $25^{\circ} \mathrm{C}$ les DLE sont égales ou supérieures à 120 min pour tous les sujets (Fig. 2) : le risque hyperthermique est quasi inexistant notamment pour le seuil d'augmentation de Tre de $1,5^{\circ} \mathrm{C}$. Le facteur physiologique limitant sera dans ce cas la déshydratation dont le niveau a pu être évalué à 10,7 $\pm 2,1 \mathrm{~g} / \mathrm{min}$ (Fig. 3) dans nos conditions expérimentales (sans réhydratation) correspondant à un déficit hydrique d'environ $640 \mathrm{~g} / \mathrm{h}$ et un niveau de déshydratation de 1,60 $\pm 0,20 \% \mathrm{du}$ poids corporel. Le niveau de fatigue perçue est à 10,8 $\pm 2,2$. 


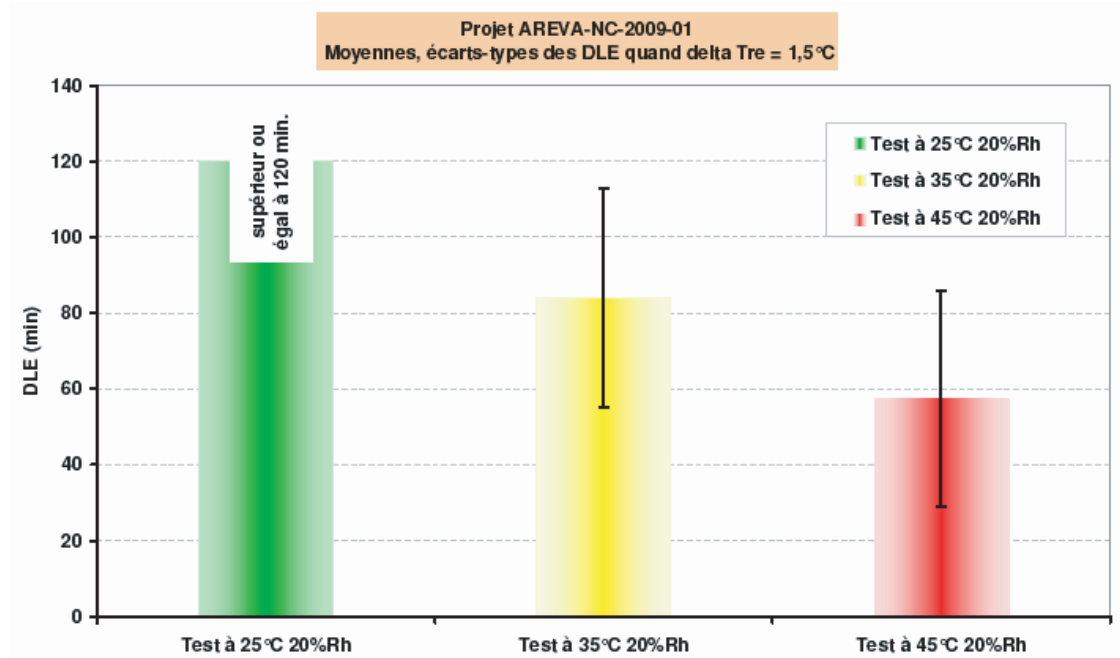

Figure 2 - Histogramme des DLE moyennes observées pour un delta de température rectale de $1,5^{\circ} \mathrm{C}$ au cours des tests.

Results of exposition time for $1.5^{\circ} \mathrm{C}$ during the tests.

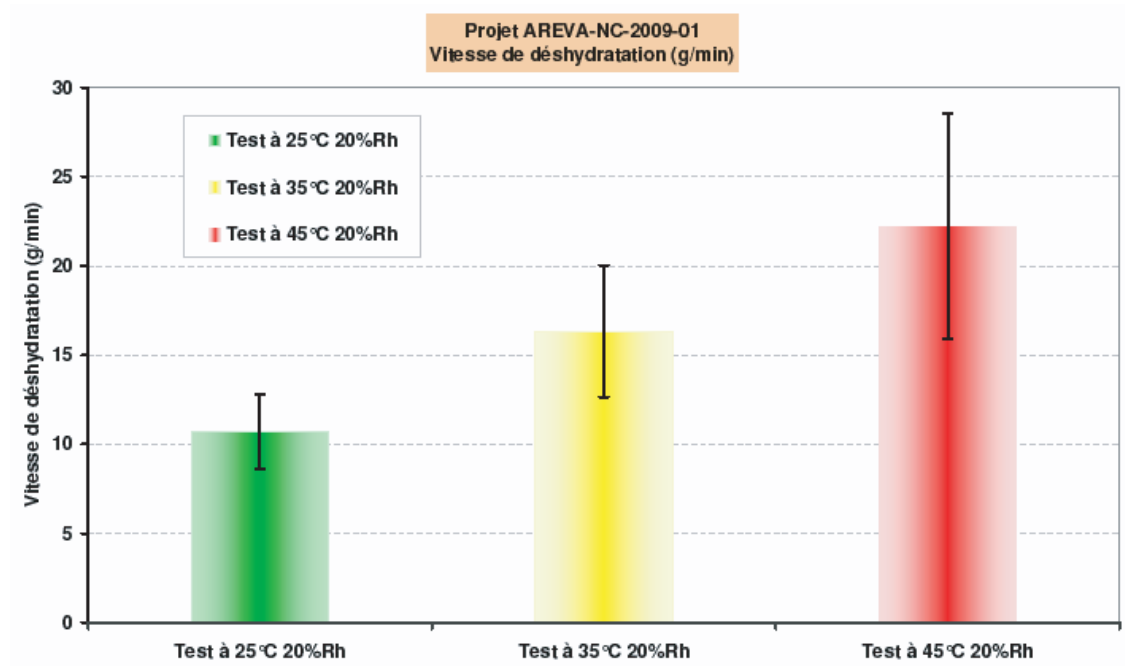

Figure 3 - Histogramme des vitesses de déshydratation moyennes au cours des tests.

Results of deshydration velocity during the tests. 


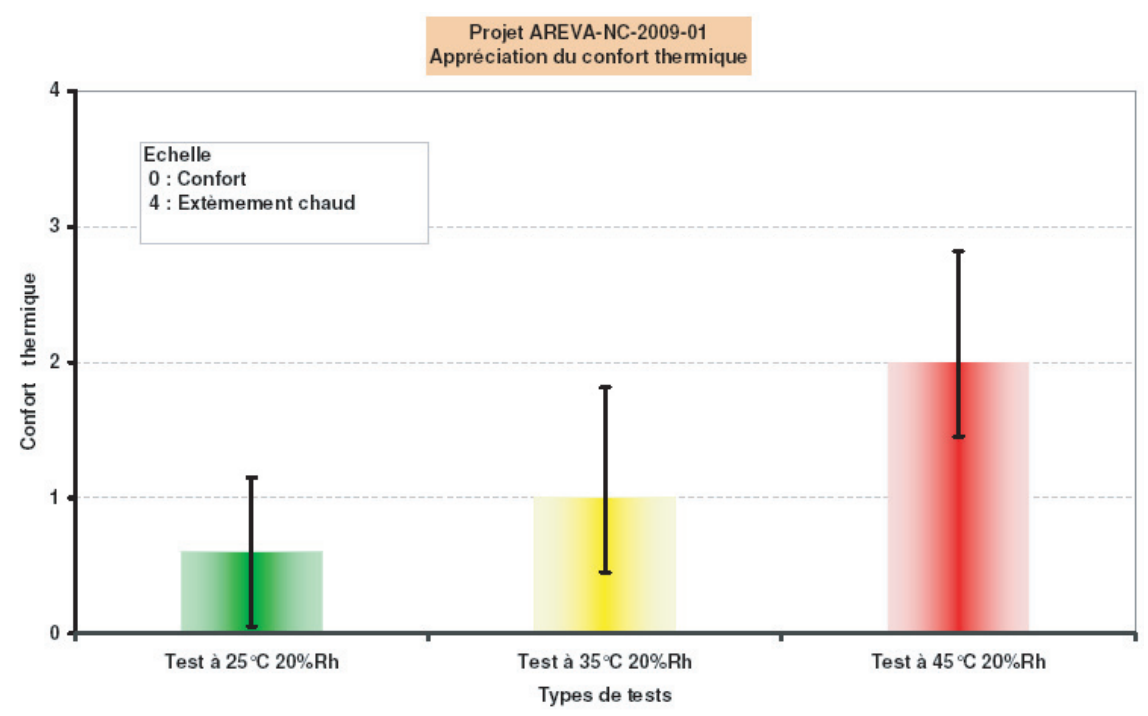

Figure 4 - Histogramme de l'appréciation moyenne du confort thermique au cours des tests. Results of thermic comfort during the tests.

\subsection{Les tests à $35^{\circ} \mathrm{C}$}

À $35^{\circ} \mathrm{C}$, la contrainte thermique n'est plus compensée totalement par le système de ventilation et le stockage thermique augmente $\left(42,2 \pm 22,9 \mathrm{~W} / \mathrm{m}^{2}\right)$. Cette contrainte se traduit par une Tre et une Tsk plus élevée en fin de test $(38,7 \pm 0,4$ et $37,0 \pm 0,3{ }^{\circ} \mathrm{C}$, respectivement) et une plus grande augmentation de la FC (144 $\pm 12 \mathrm{bpm})$.

Une grande variabilité individuelle est observée car deux sujets ont terminé les 120 min prévues pour le test et 3 sujets ont été arrêtés prématurément par les expérimentateurs du fait de l'atteinte des seuils de Tre $=39^{\circ} \mathrm{C}$. La DLE moyenne est de $96,8 \pm 28,8 \mathrm{~min}$ pour le seuil de Tre $=39^{\circ} \mathrm{C}$ et de $84 \pm 38,7 \mathrm{~min}$ pour le seuil d'augmentation de Tre de $1,5^{\circ} \mathrm{C}$ (Fig. 2). Le niveau de déshydratation a été évalué à 16,3 $\pm 3,7 \mathrm{~g} / \mathrm{min}$ (Fig. 3) dans nos conditions expérimentales, soit un déficit hydrique de l'ordre de $978 \mathrm{~g} / \mathrm{h}$ correspondant à un niveau de déshydratation de $2,0 \pm 0,8 \%$ du poids corporel.

L'inconfort thermique est ressenti comme faible (1,3 $\pm 0,9)$ (Fig. 4) et le niveau de fatigue perçue est un peu plus élevé qu'à $25^{\circ} \mathrm{C}: 11,6 \pm 2,1$. 


\subsection{Les tests à $45^{\circ} \mathrm{C}$}

À $45^{\circ} \mathrm{C}$, le stockage thermique est important $\left(63,7 \pm 29,4 \mathrm{~W} / \mathrm{m}^{2}\right)$ et l'ensemble des sujets n'a pas réalisé la durée prévue des tests car ils ont été arrêtés par les expérimentateurs du fait de l'atteinte du seuil de Tre de $39^{\circ} \mathrm{C}$, traduisant une importante contrainte thermique. La $\overline{\mathrm{T}}$ sk finale est aussi plus élevée que dans les conditions précédentes $\left(37,7 \pm 0,5^{\circ} \mathrm{C}\right)$ traduisant une thermolyse insuffisante. $\mathrm{La}$ fréquence cardiaque en fin de test est comparable à celle des tests à $35^{\circ} \mathrm{C}(144 \pm$ $13 \mathrm{bpm}$ ) mais atteinte 30 minutes plus tôt ; elle est le signe d'une contrainte cardiocirculatoire d'autant plus importante.

Ainsi, les DLE moyennes sont de $69,25 \pm 28,3$ min pour le seuil de Tre $=39^{\circ} \mathrm{C}$ et de $57,3 \pm 21,1 \mathrm{~min}$ pour le seuil d'augmentation de Tre de $1,5^{\circ} \mathrm{C}$ (Fig. 2). Le niveau de déshydratation a été évalué à $22,2 \pm 6,3 \mathrm{~g} / \mathrm{min}$ (Fig. 3) soit $1332 \mathrm{~g} / \mathrm{h}$ dans nos conditions expérimentales. Le niveau de déshydratation atteint $1,91 \pm 1,0 \%$ du poids corporel soit un niveau équivalent à celui en fin de test à $35^{\circ} \mathrm{C}$ mais atteint là aussi 30 minutes plus tôt. L'inconfort thermique est plus élevé qu'à $35^{\circ} \mathrm{C}$ $(1,9 \pm 0,7)$ (Fig. 4) ainsi que la fatigue perçue $(12,4 \pm 1,5)$.

\subsection{Les thermogrammes}

Sur les thermogrammes, les zones les plus chaudes sont situées au niveau des épaules pour le test à $45^{\circ} \mathrm{C}$. Les pieds apparaissent chauds, notamment à $45^{\circ} \mathrm{C}$ du fait de la conductance thermique liée au tapis roulant (Image IR $\mathrm{n}^{\circ} 2$ ). Ainsi, de manière globale, la distribution en air ventilé apparaît en thermographie très satisfaisante, en particulier en ce qui concerne la cagoule (Image IR $n^{\circ} 1$ ).

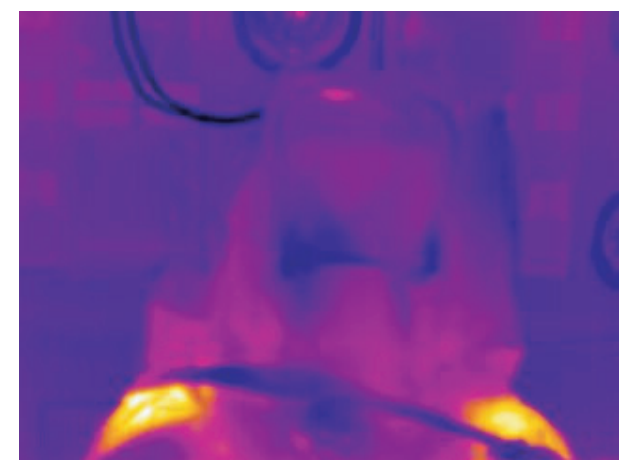

Image IR $\mathrm{n}^{\circ} 1$ - Image infrarouge de la tête : points chauds au niveau des épaules à $45^{\circ} \mathrm{C}$. Infrared picture of head: hot points in shoulders at $45^{\circ} \mathrm{C}$. 


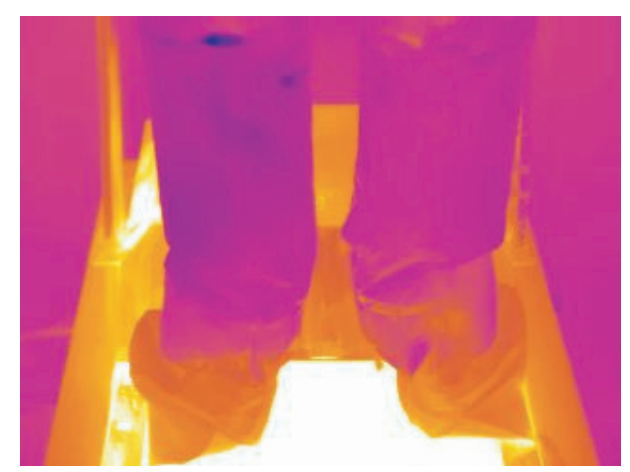

Image IR $\mathrm{n}^{\circ} 2$ - Image infrarouge des pieds (test à $45^{\circ} \mathrm{C}$ ).

Infrared picture of feet (test at $45^{\circ} \mathrm{C}$ ).

\section{Discussion}

Cette étude démontre la nécessité d'une assistance thermique par air ventilé pour prévenir les risques thermophysiologiques lors du travail de décontamination de sites nucléaires en tenue étanche.

La ventilation doit être faite selon les recommandations du constructeur en ce qui concerne le débit, la pression d'air et sa température pour assurer l'efficacité du refroidissement comme l'ont montré des essais réalisés en débit d'air limité. En effet, avec un débit de $250 \mathrm{NL} / \mathrm{min}$ au lieu de $500 \mathrm{NL} / \mathrm{min}$ au minimum, la DLE pour le seuil de Tre $=39^{\circ} \mathrm{C}$ est réduite à $81 \pm 14 \mathrm{~min}$ pour le test à $35^{\circ} \mathrm{C}$ et à $60 \pm 7$ min pour le test à $45^{\circ} \mathrm{C}$ (au lieu de $96,8 \pm 28,8$ min et $69,25 \pm 28,3 \mathrm{~min}$, respectivement). De plus, les niveaux de FC et de $\overline{\mathrm{T}} \mathrm{sk}$ sont plus élevés en fin de test. Ceci montre l'importance du respect du débit d'air minimal à la pression recommandée, la nécessité des alarmes sur la centrale de production d'air et l'intérêt d'un indicateur d'alimentation intégré à la tenue ainsi que des bouteilles de secours pour permettre le dégagement rapide éventuel.

Il faut noter que la variabilité individuelle augmente avec la température ambiante des tests et que les températures corporelles ne se stabilisent que pour le test à $25^{\circ} \mathrm{C}$. À 35 et $45^{\circ} \mathrm{C}$, la dérive des températures cutanées, de la Tre et de la fréquence cardiaque est le résultat d'une thermolyse insuffisante associée à la déshydratation. Il apparaît ainsi que la contrainte thermophysiologique dépend essentiellement de l'environnement climatique toutes choses égales par ailleurs : dépense énergétique comprise entre 30 et $38 \%$ de la puissance maximale aérobie, équipement identique, ... 
Une fois la DLE atteinte, il faut prévoir un temps de repos dans un local à température confortable $\left(25{ }^{\circ} \mathrm{C}\right.$, air sec); ce temps de repos permettra la récupération thermique et cardiaque et sera mis à profit pour une réhydratation à raison de $300 \mathrm{~mL}$ d'eau tous les 15-20 minutes ce qui devrait compenser jusqu'à trois quarts des pertes hydriques (Savourey et al., 2003 ; Melin, 2001).

\section{Conclusion}

Cette étude a permis de déterminer des DLE pour le port de la tenue TIVA dans 3 conditions climatiques lors d'un exercice physique en chambre climatique. Cet exercice effectué à puissance constante ne modélisait qu'un seul type d'exercice. A contrario du laboratoire où les conditions peuvent être précisément définies, sur le terrain la puissance du travail ne peut être constante et déterminée avec précision et les conditions climatiques variables. De plus, la tenue ne permet pas une réhydratation en cours d'effort. C'est la raison pour laquelle, dans le but de préserver la santé et la sécurité des travailleurs, les recommandations aux médecins du travail en installations nucléaires de base pour définir une limite de sécurité, pour un travail d'intensité moyenne, pourraient être les suivantes, pour une journée de travail de huit heures :

- à $25^{\circ} \mathrm{C}$, la DLE ne devra pas excéder deux séquences de travail de $120 \mathrm{~min}$ avec un temps de repos et de réhydratation de 3 heures entre les deux séquences,

- à $35^{\circ} \mathrm{C}$, la DLE ne devra pas excéder une séquence de travail de $60 \mathrm{~min}$,

- à $45^{\circ} \mathrm{C}$, la DLE ne devra pas excéder une séquence de travail de $30 \mathrm{~min}$.

\section{RÉFÉRENCES}

Borg G., Dahlstrom H. (1962) A pilot study of perceived exertion and physical working capacity, Acta Soc. Med. Ups. 67, 21-27.

Colin J., Houdas Y. (1965) Respective influence of peripheral and central thermodetectors in triggering of thermal sudation in humans, J. Physiol. (Paris) 57, 234-235.

ISO 8996 (2004) International standard organisation ergonomie de l'environnement thermique Détermination du métabolisme énergétique. Ergonomie de l'environnement thermique, Genève, CH: ISO, p. 25.

ISO 9886 (2004) International standard organisation ergonomie - Évaluation de l'astreinte thermique par mesures physiologiques. Ergonomie, Genève, CH: ISO, p. 22.

ISO 7933 (2004) International standard organisation ergonomie des ambiances thermiques Détermination analytique et interprétation de la contrainte thermique fondées sur le calcul de l'astreinte thermique prévisible. Ergonomie des ambiances thermiques, Genève, CH: ISO, p. 34.

Melin B. (2001) Eau et Sport (sportifs et sujets à activité physique intense), Chap. 14, dans : Apports nutritionnels conseillés pour la population française (A. Martin, ed) pp. 364-368. Tec and Doc, Paris, $3^{\mathrm{e}}$ édition.

Pandolf K.B., Givoni B., Goldman R.F. (1977) Predicting energy expenditure with loads while standing or walking very slowly, J. Appl. Physiol. 43(4), 577-581.

Savourey G., Launay J.C., Melin B. (2003) Physiopathologie et prévention des troubles liés à l'exposition à la chaleur ou au froid, Actualité et dossier en santé publique (ADSP), Déc. 2003, pp. 4-9. 